\title{
Characterization of a Salmonella typhimurium Mutant Defective in Phosphoribosylpyrophosphate Synthetase
}

\author{
By BJARNE U. JOCHIMSEN, ${ }^{1} \dagger$ BJARNE HOVE-JENSEN, ${ }^{1}$ \\ BRUCE B. GARBER ${ }^{2}$ AND JOSEPH S. GOTS ${ }^{2 *}$ \\ ${ }^{1}$ Enzyme Division, University Institute of Biological Chemistry B, Solvgade 83, \\ DK-1307 Copenhagen, Denmark \\ 2 Department of Microbiology, School of Medicine, University of Pennsylvania, Philadelphia, \\ Pennsylvania 19104, USA
}

(Received 2 April 1984; revised 26 June 1984)

\begin{abstract}
This study describes the isolation and characterization of a mutant (strain GP122) of Salmonella typhimurium with a partial deficiency of phosphoribosylpyrophosphate (PRPP) synthetase activity. This strain was isolated in a purE deoD gpt purine auxotroph by a procedure designed to select guanosine-utilizing mutants. Strain GP122 had roughly $15 \%$ of the PRPP synthetase activity and $25 \%$ of the PRPP pool of its parent strain. The mutant exhibited many of the predicted consequences of a decreased PRPP pool and a defective PRPP synthetase enzyme, including: (1) poor growth on purine bases; (2) decreased accumulation of 5-aminoimidazole ribonucleotide (the substrate of the blocked purE reaction) under conditions of purine starvation; (3) excretion of anthranilic acid when grown in medium lacking tryptophan; (4) increased resistance to inhibition by 5-fluorouracil; (5) derepressed levels of aspartate transcarbamylase and orotate phosphoribosyltransferase, enzymes involved in the pyrimidine de novo biosynthetic pathway;(6) growth stimulation by PRPP-sparing compounds (e.g. guanosine, histidine); (7) poor growth in low phosphate medium; and (8) increased heat lability of the defective enzyme. This mutant strain also had increased levels of guanosine 5 -monophosphate reductase. This genetic lesion, designated prs, was mapped by conjugation and phage P22-mediated transduction at 35 units on the Salmonella linkage map.
\end{abstract}

\section{INTRODUCTION}

Phosphoribosylpyrophosphate (PRPP) synthetase (ATP:D-ribose-5-phosphate pyrophosphotransferase, EC 2.7.6.1) is the enzyme which synthesizes PRPP from ribose 5-phosphate and ATP. This is a key reaction in which carbon is withdrawn from the pentose phosphate cycle and then utilized in the initial step of a highly branched biosynthetic network. PRPP is an essential precursor in the de novo biosynthesis of purine, pyrimidine and pyridine nucleotides, histidine and tryptophan. PRPP is also required for the salvage of preformed purine and pyrimidine bases and their analogues.

The activity and synthesis of PRPP synthetase in Salmonella is modulated primarily in response to the intracellular levels of various purine and pyrimidine derivatives. The pool size of uridine compounds is inversely related to the amount of enzyme synthesized so that starvation for uridine compounds results in derepression of the synthesis of the enzyme (Olszowy \& Switzer, 1972). Purine and pyrimidine nucleotides, especially adenosine 5 '-diphosphate (ADP), directly inhibit the activity of PRPP synthetase (Switzer \& Sogin, 1973). Variations in the

$\dagger$ Present address: Department of Molecular Biology and Plant Physiology, University of Aarhus, Denmark. Abbreviations: AIR, 5-aminoimidazole nucleotide; PRPP, phosphoribosyl pyrophosphate. 


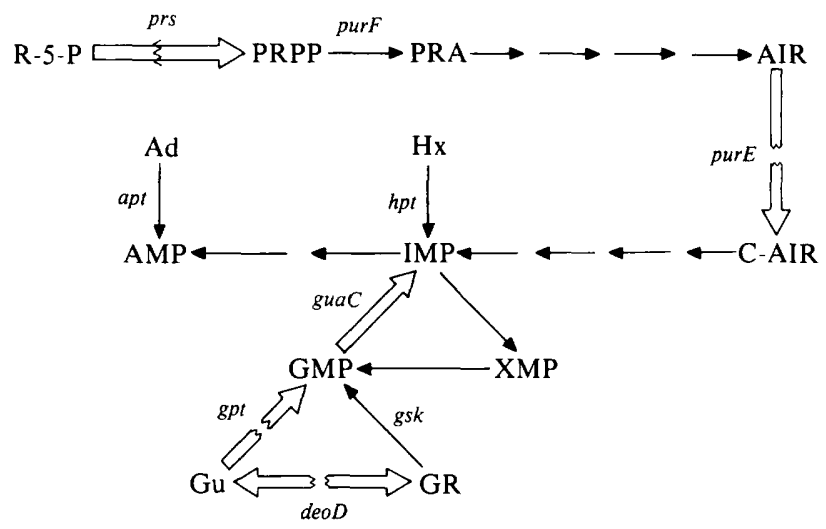

Fig. 1. Reactions of purine metabolism relevant to this study. The parent strain (GP74) is defective in the reactions indicated by broken arrows (i.e. purE, deoD, gpt). The mutant derivative strain (GP122) is in addition defective in PRPP synthetase, prs (half-broken arrow). Other gene designations: gsk, guanosine kinase; guaC, GMP reductase; apt, adenine phosphoribosyltransferase; $h p t$, hypoxanthine phosphoribosyltransferase; purF, glutamine : PRPP amidotransferase. Abbreviations: R-5-P, ribose 5phosphate; PRPP, 5-phosphoribosyl- $\alpha$-1-pyrophosphate; PRA, phosphoribosylamine; AIR, 5aminoimidazole ribonucleotide; C-AIR, 5-aminoimidazole-4-carboxylic acid ribonucleotide; IMP, inosine 5'-monophosphate; XMP, xanthosine 5'-monophosphate; Gu, guanine; GR, guanosine; Ad, adenine; Hx, hypoxanthine.

\section{Table 1. Bacterial strains}

Strain*

GP74

GP122

$\mathrm{HJ} 7$

HJ16

HJ17

HJ18

HJ74

HJ 122

JH100

KR50

SA773

SA828

\section{Genotype}

purE66 $\triangle($ proAB47-gpt) deoD28

purE66 $\triangle($ proAB47-gpt) deoD28 prs- $I$

HfrK25 serA15 zdd: :Tn $10 \triangle$ pyrF 146

purE1522 deoD203 his zdd:: Tn $10 \triangle$ pyrF 146

purE1522 deoD203 his trpE4

purE1522 deoD203 his trpE4 hemA

$\triangle($ proAB47-gpt $)$ deoD28

$\triangle($ proAB47-gpt $)$ deoD28 prs-1

purE1522 depD203 his

zdd : :Tn $10 \Delta$ pyrF 146

hemAll trpE4 proA24 met-365 pyrD197 purB210

HfrK $25 \operatorname{ser} A 15$
Source/derivation $\dagger$

Garber et al. (1980)

This study, from GP74

SA828, with P22 (KR50), Tc ${ }^{R}$

$\mathrm{JH} 100$, as $\mathrm{HJ} 7$

HJ16, with P22 (SA773), $\mathrm{Pyr}^{+}$

HJ17, neomycin-resistant

Pur $^{+}$transductant of GP74

Pur ${ }^{+}$transductant of GP122

Hoffmeyer \& Neuhard (1971)

R. Kelln

Sasarman et al. (1970)

K. E. Sanderson

* All strains are derivatives of $S$. typhimurium LT2, except strain GP74 and its derivatives (GP122, HJ74 and HJ122) whose ancestral parent was wild-type strain LT7.

$\dagger$ †. Kelln, University of Regina, Canada; K. E. Sanderson, University of Alberta, Calgary, Canada.

concentrations of histidine and tryptophan have little effect on the enzyme activity or synthesis (White et al., 1971).

This paper reports the fortuitous isolation, by a unique approach, of a mutant partially defective for PRPP synthetase activity, and the characterization of this mutant. This approach involved the selection of guanosine-utilizing mutants in a purE deoD gpt strain, a purine auxotroph which ordinarily cannot use guanosine for growth (see Fig. 1). A preliminary report of some of the data in this study has been presented (Jochimsen et al., 1980).

\section{METHODS}

Bacterial strains. All strains used are derivatives of Salmonella typhimurium LT2 or LT7, and are described in Table 1.

Media and culture techniques. Either AB medium (Monod et al., 1951), E medium (Vogel \& Bonner, 1956), or nutrient broth (Difco) was used. Amino acid and $\delta$-aminolaevulinic acid requirements were fulfilled by the appropriate compound at a concentration of $40 \mu \mathrm{g} \mathrm{ml}^{-1}$. Thiamin $\left(1 \mu \mathrm{g} \mathrm{ml}^{-1}\right)$ was routinely added to all growth media. 
Purine and pyrimidine bases were added to a final concentration of $15 \mu \mathrm{g} \mathrm{ml}^{-1}$, and nucleosides to $30 \mu \mathrm{g} \mathrm{ml}^{-1}$. Cultures were incubated at $37^{\circ} \mathrm{C}$ in a rotary shaker waterbath. Turbidity of liquid media was followed either with an Eppendorf photometer, at $436 \mathrm{~nm}$, or in a Klett-Summerson photocolorimeter with a green no. 54 filter.

Preparation of bacterial cell extracts. Bacteria that were growing exponentially were harvested by centrifugation, and washed twice with $0.9 \% \mathrm{NaCl}$. Cells from $100 \mathrm{ml}$ of culture were resuspended in $1 \mathrm{ml}$ of sonication buffer ( $50 \mathrm{~mm}$-potassium phosphate, $\mathrm{pH} 8.0$, containing $50 \mathrm{mM}$-triethanolamine and $0.75 \mathrm{mM}$-EDTA). The concentrated cells were sonically disrupted with an MSE ultrasonic disintegrator for $60 \mathrm{~s}$ at $0{ }^{\circ} \mathrm{C}$, and cell debris was removed by centrifugation. For determination of the activity of aspartate transcarbamylase, GMP reductase, and orotate phosphoribosyltransferase the supernatant was used without further treatment. For determination of PRPP synthetase activity, the extract was passed through a small Sephadex G25 column equilibrated with $50 \mathrm{~mm}$-potassium phosphate buffer, $\mathrm{pH} 7.5$.

Enzyme assays. Aspartate transcarbamylase and orotate phosphoribosyltransferase were assayed as described by Schwartz \& Neuhard (1975). GMP reductase was measured as described by Benson et al. (1971). PRPP synthetase was assayed as described by Switzer (1969) with the modifications of Jensen $e t$ al. (1979). Specific activity is defined as nmol of substrate consumed, or product formed per min per mg protein at $37^{\circ} \mathrm{C}$.

Biochemical assays. Protein concentrations were determined by the Lowry method with bovine serum albumin as the standard. Aminoimidazole ribonucleotide (and derivatives) and anthranilic acid were assayed as diazotizable arylamines by the method of Bratton \& Marshall (1939).

Nucleoside triphosphate and PRPP pool determinations. Cells growing exponentially in Tris-buffered minimal medium (Irr \& Gallant, 1969) containing $1 \mathrm{mM}$-phosphate, were labelled with $\left.{ }^{32} \mathrm{P}\right]$ orthophosphate [33 $\mathrm{Ci} \mathrm{mol}^{-1}$ (1221 GBq mol ${ }^{-1}$ ); Atomenergikommissionens Forsøgsanlaeg, Risø, Denmark]. Extraction, two-dimensional thin-layer chromatography, identification, and counting of radioactive compounds were done as described by Jensen et al. (1979).

Genetical methods. Uninterrupted conjugations were done as described by Miller (1972). Transductions using P22, L3 (Beck \& Ingraham, 1971) or P22HT105/int201 (Anderson \& Roth, 1978) have been described previously. $\mathrm{Hem}^{+}$transductants were selected on nutrient agar. Tetracycline resistance $\left(\mathrm{Tc}^{\mathrm{R}}\right)$ was selected on minimal medium supplemented with tetracycline $\left(10 \mu \mathrm{g} \mathrm{ml}^{-1}\right)$.

Isolation of PRPP synthetase mutant. An exponential phase culture of strain GP74, growing in nutrient broth was concentrated from $10 \mathrm{ml}$ to $0.5 \mathrm{ml}$ and $0.1 \mathrm{ml}$ was plated on minimal salts/glucose agar plates containing guanosine $\left(50 \mu \mathrm{g} \mathrm{ml}^{-1}\right)$, proline $\left(100 \mu \mathrm{g} \mathrm{ml}^{-1}\right)$, and thiamin $\left(1 \mu \mathrm{g} \mathrm{ml}^{-1}\right)$. Colonies that appeared after $2 \mathrm{~d}$ incubation were picked and purified on identical plates. Pur ${ }^{+}$revertants were discarded. A colony of each guanosine-utilizing mutant was then inoculated into $2 \mathrm{ml}$ minimal salts/glucose medium containing hypoxanthine ( $\left.2 \mu \mathrm{g} \mathrm{ml}^{-1}\right)$, casein hydrolysate $(0.1 \%)$ and thiamin $\left(1 \mu \mathrm{g} \mathrm{ml}^{-1}\right)$. The cells were incubated for $18 \mathrm{~h}$, pelleted, and the supernatants were tested for 5-aminoimidazole ribonucleoside. Mutants with decreased or absent accumulation of this compound were then used for phage P22-mediated recombinational analysis with purine auxotrophs blocked prior to the purE reaction (i.e. $p u r F, p u r D, p u r G$ and $p u r I$ ).

\section{RESULTS}

One mutant (GP122) selected as described in Methods grew well on guanosine as sole purine source, and accumulated 5-aminoimidazole ribonucleotide (AIR) in decreased amounts during the crude $18 \mathrm{~h}$ accumulation assay. The kinetics of AIR accumulation were studied in more detail (Fig. 2) and it was found that the rate of AIR accumulation by the mutant was only $24 \%$ of that of the parent strain, under identical conditions of purine starvation. From this we inferred that strain GP122 had acquired an additional lesion in the purine de novo biosynthetic pathway prior to the purE reaction.

However, strain GP122 was found to be non-allelic with any of the four known early-blocked pur mutants (see Methods), as evidenced by normal prototrophic recombinational frequencies. We then considered the possibility that the defect involved PRPP synthetase. This enzyme catalyses the formation of a substrate required for the de novo biosynthesis of purine nucleotides, and the reaction might thus be considered an extension of the classical purine pathway (Fig. 1). PRPP synthetase activity was measured in strains GP74 and GP122 (Table 2), and the mutant was found to have only $15-23 \%$ of the activity of the parent strain. In addition, PRPP synthetase from strain GP122 was extremely heat-labile during in vitro incubation, as compared to the enzyme from its parent strain GP74 (Fig. 3), indicating that the enzyme itself is altered and that the mutation is probably within the structural gene for PRPP synthetase. One additional pertinent finding was that strain GP122 had elevated, but not fully constitutive levels of GMP reductase 


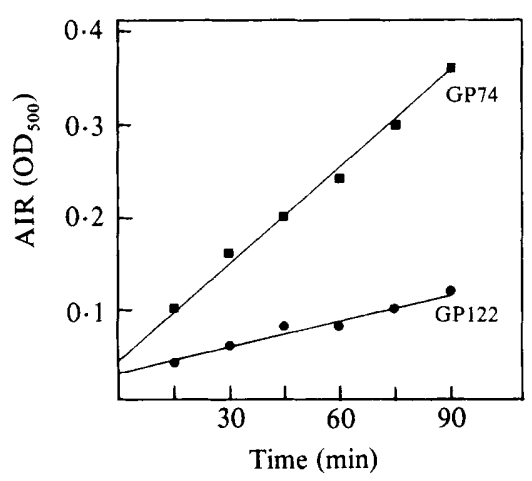

Fig. 2

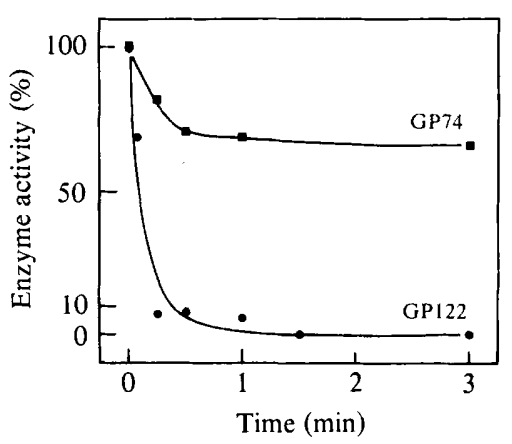

Fig. 3

Fig. 2. Accumulation of 5-aminoimidazole ribonucleotide (AIR) by resting suspensions of strains GP74 $\left(\right.$ prs $\left.^{+}\right)$and GP122 (prs). Cultures were grown overnight in minimal salts/glucose medium supplemented with proline, thiamin and hypoxanthine. The cells were harvested by centrifugation, washed with saline, resuspended at a concentration of $0.5 \mathrm{mg}^{2}$ dry wt ml-1 in purine-free minimal salts/glucose medium containing $0.1 \%$ casein hydrolysate, and $25 \mu \mathrm{g}$ tetracycline $\mathrm{ml}^{-1}$ (to prevent derepression of enzyme synthesis). Incubation was at $37^{\circ} \mathrm{C}$ with shaking. Samples were withdrawn at various times and acetylated with acetic anhydride (to remove interfering arylamines). AIR (excreted as the ribonucleoside) was detected by the Bratton-Marshall (1939) reaction and the absorbance of the resulting orange chromophore was measured at $500 \mathrm{~nm}$.

Fig. 3. Heat inactivation of PRPP synthetase from strains GP74 $\left(\right.$ prs $\left.^{+}\right)$and GP122 (prs). Cell-free extracts were prepared as described in Methods. At zero time extracts were heated to $56^{\circ} \mathrm{C}$. Samples were removed at intervals, cooled on ice, and the residual enzyme activity was assayed. Heat inactivation was done at a protein concentration of $5 \mathrm{mg} \mathrm{ml}^{-1}$ in $50 \mathrm{mM}$-potassium phosphate buffer, pH 7.5. The enzyme activities corresponding to $100 \%$ were those shown in Table 2.

\section{Table 2. PRPP synthetase and GMP reductase activity in strains GP74 and GP122}

Cells were grown in minimal salts/glucose medium supplemented with guanosine (GR), adenine (Ad), histidine (His) or hypoxanthine ( $\mathrm{Hx})$; NG, no growth. Results are nmol $\mathrm{min}^{-1}(\mathrm{mg} \text { protein) })^{-1}$. The values were reproducible within $10 \%$ in repeated experiments.

$\begin{array}{lcccr}\text { Strain } & \begin{array}{c}\text { PRPP } \\ \text { synthetase } \\ \text { activity }\end{array} & \overbrace{\text { GR }} & \text { Ad + GR + His } & \text { Hx } \\ \text { GP74 } & 58 & \text { NG } & 1 & 3 \\ \text { GP122 } & 9 & 15 & 8 & 12\end{array}$

under all growth conditions tested (Table 2). Elevated reductase levels are known to facilitate guanosine utilization (Gots et al., 1977; Garber et al., 1980).

\section{PRPP and nucleoside triphosphate pools}

Decreased levels of PRPP synthetase in strain GP122 would be expected to alter the intracellular pool of PRPP and eventually the purine and pyrimidine nucleotide pools. To document this, nucleoside triphosphate and PRPP pools were measured in $p u r E^{+}$derivatives of strains GP74 and GP122 (Table 3). Purine independent derivatives were used so that pools could be measured in the absence of exogenous purines. Strain HJ122 (prs purE $E^{+}$) had lowered levels of purine nucleoside triphosphates, and its PRPP pool was less than $25 \%$ of that in the parent strain $\mathrm{HJ} 74$ (prs purE ${ }^{+}$), confirming our predictions.

\section{Effects of lowered PRPP pool on the physiology of strain GP122}

The finding of a lowered PRPP pool in strain GP122 prompted us to study the effect on growth of adding PRPP-sparing compounds to the growth medium (Table 4). Some of these 
Table 3. Nucleoside triphosphate and PRPP pools in prs and prs ${ }^{+}$strains

Purine prototrophic strains were grown without purine supplementation in glucose minimal medium. Pools were determined as described in Methods. The values are nmol mg dry wt ${ }^{-1}$ and showed less than $10 \%$ variation in duplicate experiments.

\begin{tabular}{lcccccc}
\multirow{2}{*}{ Strain } & $\begin{array}{c}\text { prs } \\
\text { allele }\end{array}$ & $\overbrace{\text { PRPP }}$ & ATP & GTP & CTP & UTP \\
HJ74 & + & 2.1 & 2.7 & 1.6 & 0.6 & 0.8 \\
HJ122 & - & 0.5 & 2.4 & 1.2 & 0.7 & 1.1
\end{tabular}

Table 4. Effect of PRPP-sparing compounds on the growth of prs and prs ${ }^{+}$strains

\begin{tabular}{|c|c|c|}
\hline \multirow[b]{2}{*}{ Medium* } & \multicolumn{2}{|c|}{ Generation time ( $\mathrm{min})$} \\
\hline & $\begin{array}{c}\text { Strain GP74 } \\
\text { prs }^{+}\end{array}$ & $\begin{array}{c}\text { Strain GP122 } \\
\text { prs }\end{array}$ \\
\hline $\begin{array}{l}\text { A High phosphate } \\
\text { plus uracil } \\
\text { plus tryptophan and histidine } \\
\text { plus adenine and histidine } \\
\text { plus guanosine }\end{array}$ & $\begin{array}{l}52 \\
50 \\
51 \\
48 \\
49\end{array}$ & $\begin{array}{r}101 \\
103 \\
82 \\
77 \\
60\end{array}$ \\
\hline B Low phosphate & 53 & 320 \\
\hline
\end{tabular}

* Medium A, minimal salts/glucose (phosphate concentration $50 \mathrm{~mm}$ ), hypoxanthine, proline and thiamin; medium B, same as A, but phosphate concentration $1.0 \mathrm{~mm}$.

compounds increased the growth rate of strain GP122. A reduction in the phosphate concentration of the medium from $50 \mathrm{mM}$ to $1 \mathrm{~mm}$ drastically reduced the growth rate (Table 4). This is in accord with the known requirement of PRPP synthetase for inorganic phosphate as a co-factor and as a stabilizing agent (Switzer, 1969). It is possible that the defective PRPP synthetase enzyme is even more dependent on phosphate than is the normal enzyme.

While studying the kinetics of AIR accumulation in growing cultures, another arylamine was found. This differed from AIR by being acetylable, by the absorption spectrum of its purple chromophore generated via the Bratton-Marshall reaction (maximum at $550 \mathrm{~nm}$, as compared with $500 \mathrm{~nm}$ maximum of the orange AIR chromophore), and by its continued accumulation in medium containing high purine concentrations (which repress the accumulation of AIR). This new arylamine was identified as anthranilic acid on the basis of its UV absorption spectrum, its characteristic blue fluorescence, its chromatographic identity with a known sample of anthranilic acid, its ability to feed $\operatorname{trp} E$ (anthranilate synthetase) but not $\operatorname{trp} A, B, C$ or $D$ mutants, and because exogenous tryptophan prevented its accumulation (Fig. 4 illustrates this latter effect). The accumulation of anthranilate by this mutant is consistent with the concept that the low intracellular PRPP pool causes the anthranilate phosphoribosyltransferase reaction to proceed at a greatly lowered rate. The addition of tryptophan alone had no effect on the generation time of strain GP122. This might indicate that the trp operon is derepressed to an extent that can compensate for the deficiency of PRPP, and hence maintain the requisite rate of tryptophan synthesis. The parent strain GP74 did not accumulate anthranilate.

Another finding which might be expected in strain GP122 is altered resistance to inhibition by purine or pyrimidine analogues. It is well established that such analogues are usually not inhibitory until they are converted to their corresponding nucleotide form via a phosphoribosyltransferase enzyme, and that resistance to this inhibition is correlated with loss of this ability (Kalle \& Gots, 1961, 1963). Specifically, resistance may be due to an alteration or deficiency of the appropriate phosphoribosyltransferase enzyme or, theoretically, to a shortage of a required substrate (i.e. PRPP). Strain GP122 showed a fourfold increase in the minimal inhibitory concentration of 5-fluorouracil as compared with strain GP74 $\left(0.5 \mu \mathrm{g} \mathrm{ml}^{-1} \mathrm{vs} 2.0 \mu \mathrm{g} \mathrm{ml}^{-1}\right)$. In addition, strain GP122 was found to have levels of orotate phosphoribosyltransferase and aspartate 


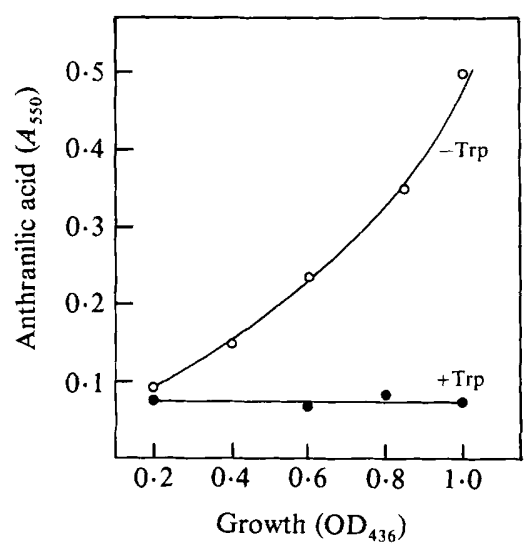

Fig. 4. Accumulation of anthranilic acid by strain GP122(prs), and prevention of this accumulation by tryptophan. Strain GP122 was inoculated into two portions of minimal salts/glucose medium containing proline, thiamin and hypoxanthine, one of which also contained tryptophan. Samples were withdrawn at various times during the exponential growth phase. Anthranilic acid was detected by the Bratton-Marshall (1939) reaction, without prior acetylation, and the absorbance of the resulting purple chromophore was measured at $550 \mathrm{~nm}$. This was plotted as a function of culture growth $\left(\mathrm{OD}_{436}\right)$. Trp, tryptophan.

Table 5. Genetic mapping of prs

Cross
no.

2

GP122 (prs)
Donor strain

HJ7 (HfrK25 ser A $z d d:: \operatorname{Tn} 10$ pyrF)
Recipient strain

GP122 (purE deoD prs)

HJ18 (purE deoD hemA)

\section{Selected}

markers

$\operatorname{Tn} 10, \operatorname{ser} A^{+}$

\section{Unselected} markers†

pyrF $F^{+}$prs

pyrF prs

pyrF prs ${ }^{+}$

pyrF ${ }^{+}$prs $^{+}$

prs $^{+}$

prs
No.

* Recombinants in cross 1 were obtained by conjugation, and those in cross 2 by P22 transduction. Selective plates contained guanosine plus hypoxanthine as sources of purines.

$\dagger$ Scoring of prs was done on medium containing guanosine as sole purine source.

transcarbamylase three and six times higher, respectively, than those in the parent strain (data not shown).

\section{Map location of the structural gene for PRPP synthetase}

For conjugational mapping strain $\mathrm{HJ} 7$ was constructed. This strain harbours a $\mathrm{Tn} 10$ insertion ( $z d d:: \operatorname{Tn} 10$ ) which co-transduces $75 \%$ (P22-mediated transduction) with pyrF (R. Kelln, personal communication). Strain $\mathrm{HJ} 7$ was crossed with strain GP122 and $\mathrm{Tc}^{\mathrm{R}}$ recombinants were selected (Table 5, cross 1 ). Among the $\mathrm{Tc}^{\mathrm{R}}$ recombinants obtained, $93 \%$ inherited the $p y r F$ allele from the donor, whereas $56 \%$ lost the ability to use guanosine as sole purine source. The gene order deduced was $z d d:: \operatorname{Tn} 10-p y r F-p r s$. Assuming an exponential decrease of transfer of distal markers (de Haan et al., 1969), the location of the guanosine growth property is 1-2 min clockwise of $z d d::$ Tn 10. To verify this, strain HJ18 (purE deoD hemA) was used as recipient and strain GP122 as transductional donor, and $\mathrm{Hem}^{+}$transductants were selected. Table 5 (cross 2 ) shows that $51 \%$ of the recombinants gained the ability to use guanosine as sole purine source, indicating acquisition of the defective PRPP synthetase.

The recombinants obtained in the crosses given in Table 5 were further examined with respect to heat-lability and specific activity of PRPP synthetase, activity of GMP reductase, accumulation of AIR, and growth in low phosphate medium. All the recombinants from cross 1 which lost the ability to use guanosine as sole purine source also inherited wild-type PRPP 
synthetase, repressed synthesis of GMP reductase when cultured with adenine and guanosine present in the growth medium, wild-type growth in low phosphate medium and wild-type accumulation of AIR. In contrast, the recombinants from cross 2 which inherited the ability to use guanosine displayed mutant properties of PRPP synthetase and GMP reductase, as well as poor growth in low phosphate medium and decreased accumulation of AIR. Thus a single mutation is responsible for these phenotypes. In keeping with a similar mutant described in Escherichia coli (Hove-Jensen, 1983) this genetic locus is now designated prs, rather than a previously designated prpP (Jochimsen et al., 1980).

\section{DISCUSSION}

The PRPP synthetase mutant described in this paper (strain GP122) was obtained fortuitously by a procedure designed to select guanosine-utilizing mutants of strain GP74. Salient characteristics of strain GP74 include: (i) accumulation of 5-aminoimidazole ribonucleotide (AIR), the substrate of the defective purE reaction, under conditions of purine starvation, and (ii) deficiency of both guanine (xanthine) phosphoribosyltransferase (gpt) and purine nucleoside phosphorylase $(d e o D)$ (see Fig. 1). The latter two enzyme deficiencies render this strain unable to use guanosine alone to supply its adenine and guanine nucleotide requirements, despite having the requisite enzymes (i.e. guanosine kinase and GMP reductase). This inability to use guanosine is thought to be due to a limitation in the rate of conversion of guanosine to GMP and thence to AMP, imposed by limiting activities of guanosine kinase and GMP reductase.

Characterization of other mutant derivatives of strain GP74 that could utilize guanosine has previously revealed several classes (Gots et al., 1977). Some had increased levels of guanosine kinase or GMP reductase as might be predicted, but an unexpected class possessed an additional defect in the purine de novo pathway prior to the purE reaction (e.g. a purF or purG lesion). With regard to this latter class of mutants, preliminary data indicate that AIR is an inhibitor of GMP reductase (unpublished observation). Therefore, purE strains that had incurred an additional lesion which prevented or decreased the accumulation of AIR (e.g. a purF, purG and purI lesion) might owe their ability to utilize guanosine to the removal of this inhibition. None of these three lesions resulted in elevated levels of GMP reductase. The reasons why a partial PRPP synthetase mutant was obtained by this selection procedure are not entirely clear, but the most likely possibilities include the following: (i) the decreased accumulation of AIR by this type of mutant (Fig. 2) would decrease any AIR-mediated inhibition of GMP reductase, similar to the situation with the purF, purG and purI mutants; and (ii) the PRPP synthetase mutant, unlike the other early de novo blocked mutants, has an elevated level of GMP reductase (Table 2), a condition which is known to facilitate guanosine utilization (Garber et al., 1980; Gots et al., 1977).

The reason for the elevated GMP reductase activity in strain GP122 is unclear at present. However, most of the other properties of this strain are the predictable consequences of a defective PRPP synthetase enzyme (e.g. increased heat lability, poor growth in low phosphate media) and of a decreased PRPP pool (e.g. excretion of anthranilic acid, increased resistance to inhibition by 5-fluorouracil, and growth stimulation by PRPP-sparing compounds).

Mapping of the PRPP synthetase structural gene prs revealed a location at 35 units on the new 100 unit Salmonella linkage map (Sanderson \& Roth, 1983), close to the hem $A$ gene.

Hove-Jensen \& Nygaard (1982) have isolated a PRPP synthetase mutant in E. coli, using the same selection procedure as in this study. This mutant has an enzyme with altered kinetic parameters, and is defective in the structural gene for PRPP synthetase. The lesion mapped very close to the hemA gene (Hove-Jensen \& Nygaard, 1982), which is at $26 \mathrm{~min}$ in E. coli. Pandey \& Switzer (1982) have also independently isolated, by a different technique, a Salmonella mutant which is defective in PRPP synthetase. The properties reported differ from those of strain GP122, and the mutation in their strain maps at a completely different locus (46 units on the new Salmonella map) than does the prs lesion. The relationship between our mutant and this other Salmonella mutant requires further clarification. 
The excellent technical assistance of J. Steno Christensen is gratefully appreciated.

This investigation was supported by grant no. CA- 02790 awarded by the National Cancer Institute, DHEW, by grant PCM 79-05753 from the National Science Foundation, and by grants from the Danish Natural Science Research Council.

\section{REFERENCES}

Anderson, R. P. \& Roth, J. R. (1978). Tandem chromosomal duplications in Salmonella typhimurium: fusion of histidine genes to novel promotors. Journal of Molecular Biology 119, 147-166.

BeCK, C. F. \& INGRAHAM, J. L. (1971). Location on the chromosome of Salmonella typhimurium of genes governing pyrimidine metabolism. Molecular and General Genetics 111, 303-316.

Benson, C. E., BREhMEYeR, B. A. \& Gots, J. S. (1971). Requirement for cyclic AMP for induction of GMP reductase in Escherichia coli. Biochemical and Biophysical Research Communications 43, 1089-1094.

Bratton, A. C. \& Marshall, E. K. (1939). A new coupling component for sulfanilamide determination. Journal of Biological Chemistry 128, 537-550.

GARBER, B. B., JoChIMSEN, B. U. \& GoTS, J. S. (1980). Glutamine and related analogs regulate guanosine monophosphate reductase in Salmonella typhimurium. Journal of Bacteriology 143, 105-111.

Gots, J. S., Benson, C. E., Jochimsen, B. U. \& Koduri, R. K. (1977). Microbial models and regulatory elements in the control of purine metabolism. In Purine and Pyrimidine Metabolism (CIBA Foundation Symposium vol. 48), pp. 23-41. Amsterdam: Elsevier/North-Holland.

de HaAn, P. G., Hoekstra, W. P. M., Verhoef, C. \& FeLIX, H. S. (1969). Recombination in Escherichia coli. III. Mapping by the gradient of transmission. Mutation Research 8, 505-512.

Hoffmeyer, J. \& Neuhard, J. (1971). Metabolism of exogenous purine bases and nucleosides by Salmonella typhimurium. Journal of Bacteriology 106, 14-24.

HOVE-JENSEN, B. (1983). Chromosomal location of the gene encoding phosphoribosylpyrophosphate synthetase in Escherichia coli. Journal of Bacteriology 154, 177-184.

Hove-Jensen, B. \& Nygaard, P. (1982). Phosphoribosylpyrophosphate synthetase of Escherichia coli. Identification of a mutant enzyme. European Journal of Biochemistry 126, 327-332.

IRR, J. \& GallaNT, J. (1969). The control of ribonucleic acid synthesis in Escherichia coli. II. Stringent control of energy metabolism. Journal of Biological Chemistry 244, 2233-2239.

Jensen, K. F., Houlberg, U. \& NygaArd, P. (1979). Thin-layer chromatographic methods to isolate 32P-labeled 5-phosphoribosyl- $\alpha-1$-pyrophosphate (PRPP): determination of cellular PRPP pools and assay of PRPP synthetase activity. Analytical Biochemistry 98, 254-263.

JoChimsen, B., GARBER, B. \& GoTs, J. S. (1980). Phosphoribosylpyrophosphate synthetase mutant in Salmonella typhimurium. Advances in Experimental Medicine and Biology 122B, 131-136.
Kalle, G. P. \& Gots, J. S. (1961). Alterations in purine nucleotide pyrophosphorylases and resistance to purine analogues. Biochimica et biophysica acta 53, 166-173.

Kalle, G. P. \& Gots, J. S. (1963). Genetic alteration of adenylic pyrophosphorylase in Salmonella. Science 142, 680-681.

MILLER, J. H. (1972). Experiments in Molecular Genetics. Cold Spring Harbor, NY: Cold Spring Harbor Laboratory.

Monod, J., Cohen-Bazire, G. \& Cohn, M. (1951). Sur la biosynthèse de la betagalactosidase (lactase) chez Escherichia coli. La spécificité de l'induction. Biochimica et biophysica acta 7, 585-590.

Olszowy, J. \& Switzer, R. L. (1972). Specific repression of PRPP synthetase by uridine compounds in Salmonella typhimurium. Journal of Bacteriology 110, 450-451.

Pandey, N. K. \& Switzer, R. L. (1982). Mutant strains of Salmonella typhimurium with defective phosphoribosylpyrophosphate synthetase activity. Journal of General Microbiology 128, 1863-1871.

SANDERSON, K. E. \& Roth, J. R. (1983). Linkage map of Salmonella typhimurium, edition VI. Microbiological Reviews 47, 410-453.

Sasarman, A., Sanderson, K. E., Surdeanu, M. \& SonEA, S. (1970). Hemin-deficient mutants of Salmonella typhimurium. Journal of Bacteriology 102, 531-536.

SchWartz, M. \& Neuhard, J. (1975). Control of expression of the pyr genes in Salmonella typhimurium: effects of variations in uridine and cytidine nucleotide pools. Journal of Bacteriology 121, 814822.

SwITZER, R. L. (1969). Regulation and mechanism of phosphoribosylpyrophosphate synthetase, I Purification and properties of the enzyme from Salmonella typhimurium. Journal of Biological Chemistry 244, 2854-2863.

Switzer, R. L. \& Sogin, D. C. (1973). Regulation and mechanism of phosphoribosylpyrophosphate synthetase. V Inhibition by end products and regulation by adenosine diphosphate. Journal of Biological Chemistry 248, 1063-1073.

Vogel, H. J. \& BonNer, D. M. (1956). Acetylornithase of Escherichia coli: partial purification and some properties. Journal of Biological Chemistry 218, 97106.

White, M. N., Olszowy, J. \& SWitzer, R. L. (1971). Regulation and mechanism of phosphoribosylpyrophosphate synthetase: repression by end products. Journal of Bacteriology 108, 122-131. 